\title{
Contribution de la biodiversité à l'éco-oenotourisme des vignobles héroïques : atouts et perspectives
}

\section{Contribution of biodiversity to the eco-oenotourism of heroic vineyards: Assets and perspectives}

\author{
J. Rochard \\ IFV (Institut Français de la Vigne et du Vin), 17 rue Jean Chandon Moët, 51200 Epernay, France
}

\begin{abstract}
Résumé. L'œnotourisme prend une part grandissante dans la valorisation des territoires viticoles. Parallèlement à l'attrait culturel pour la vigne, le vin et la dégustation, émerge le concept « d'EcoOenotourisme » qui associe notamment, en liaison avec le développement durable, les paysages, la biodiversité, ainsi que l'éco-conception des caves. Ainsi la valorisation de la biodiversité participe souvent à la valorisation du paysage local. C'est également un facteur qui participe à l'image et à la valeur ajoutée des vins de ces régions. Cette thématique est importante pour les viticulteurs qui sont sensibles au regard que la société porte sur leur métier, voire pour l'acceptation locale de leurs activités et des projets qu'ils souhaitent mettre en œuvre. Les terroirs des vignobles « héroïques 》 ont souvent su conserver les modes de production traditionnels, associés notamment à la présence de murets, talus, zones naturelles, à l'agroforesterie traditionnelle ; savoir-faire historiques, supports de spécificités patrimoniales mais également de valeurs écologiques spécifiques des terrasses ou des îles.
\end{abstract}

\begin{abstract}
Wine tourism is becoming increasingly important in the valuation of viticultural territories. In parallel with the cultural attraction for the vine, the wine and the tasting, emerges the concept of "Eco-Wine Tourism" which associates in particular, in connection with the sustainable development, the landscapes, the biodiversity, as well as the eco-design cellars. Thus the valorization of the biodiversity often contributes to the valorization of the local landscape. It is also a factor that contributes to the image and added value of wines from these regions.This theme is important for wine growers who are sensitive to the way society looks at their business, or even for the local acceptance of their activities and the projects they want to implement. The terroirs of "heroic" vineyards have often retained traditional production methods, associated in particular with the presence of low walls, embankments, natural areas, traditional agroforestry; historical know-how, support for heritage specificities but also ecological values specific terraces or islands.
\end{abstract}

\section{Introduction}

L'œnotourisme prend une part grandissante dans la valorisation des territoires viticoles. C'est également un facteur qui participe à l'image et à la valeur ajoutée des vins de ces régions [1]. Parallèlement à l'attrait culturel pour la vigne, le vin et la dégustation, l'Enotourisme est également motivé par une approche écologique des caves et des terroirs. Ainsi émerge le concept « d'écoœnotourisme » qui associe notamment, en liaison avec le développement durable, les paysages, la biodiversité, ainsi que l'éco-conception des caves [2]. Le viticulteur, au-delà de l'élaboration des vins, intègre la promotion des terroirs, la conception et l'aménagement des caves. Dans sa mission d'ambassadeur de l'Eco-œnotourisme, il doit veiller à mettre en œuvre les bases environnementales d'une viticulture et d'une œnologie durables concept, définies par une résolution de l'OIV (CST 01-2008) « Approche globale à l'échelle des systèmes de production et de transformation des raisins, associant à la fois la pérennité économique des structures et des territoires, l'obtention de produits de qualité, la prise en compte des exigences d'une viticulture de précision, des risques liés à l'environnement, à la sécurité des produits et la santé des consommateurs et la valorisation des aspects patrimoniaux, historiques, culturels, écologiques et paysagers ».

Cette thématique est importante pour les viticulteurs qui sont sensibles au regard que la société porte sur leur métier, voire pour l'acceptation de leurs activités et des projets qu'ils souhaitent mettre en œuvre, notamment lorsque des aspects conflictuels peuvent apparaître avec la population ou les associations de protections de la nature [3].

Dans certains cas, la valorisation des vins est directement associée au maintien des structures essentielles d'un paysage comme expression esthétique et patrimoniale d'un lien au terroir (pour les produits AOC par exemple) tel qu'il est défini par l'OIV (Viti 333/2010) : Le « terroir » vitivinicole est un concept qui se réfère à un espace sur lequel se développe un savoir collectif des interactions entre un milieu physique et biologique identifiable et les pratiques vitivinicoles appliquées, qui confèrent des 


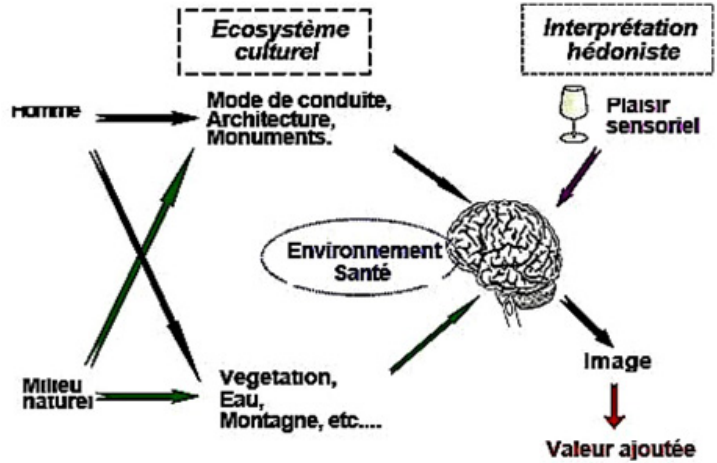

Figure 1. Composante de l'image d'un vin. (Source : J. Rochard, Traité de Viticulture et d'œnologie durable, Editions Avenir (Enologie, 2005).

caractéristiques distinctives aux produits originaires de cet espace. Le 《terroir 》 inclut des caractéristiques spécifiques du sol, de la topographie, du climat, du paysage et de la biodiversité.

Parallèlement les viticulteurs ont intérêt à offrir un paysage de qualité, support emblématique de l'activité touristique de leur région, vecteur d'image de leur métier et de leurs produits. A propos de la valeur symbolique, émotionnelle, affective du terroir, S. MICHEL précise que «Nous sommes ce que nous mangeons. La méconnaissance de ce que nous avons dans notre assiette ou notre verre conduit à une perte d'identité, mais heureusement le terroir restaure le lien entre le consommateur et l'aliment. Les valeurs symboliques de l'aliment sont celles du terroir mais attention, toute contamination du terroir ; qu'elle soit physique, chimique, biologique ou visuelle, contamine le produit dans l'esprit $d u$ client $»$. Cette réflexion souligne que le terroir, et dans son prolongement la cave, sont des atouts, supports de valorisation, mais à l'inverse une dénaturation des paysages, ou une approche peu valorisante de la cave, peuvent dégrader la perception du terroir et indirectement l'image du vin du producteur (Fig. 1) [4].

\section{Contribution paysagère des terrasses}

La viticulture, comme l'agriculture, a intégré les apports de la modernité dans la conduite de ses itinéraires techniques. A la fin du XIXe siècle, la crise phylloxérique, parallèlement au développement de la traction animale, a profondément modifié la gestion des terroirs viticoles. Puis progressivement, l'introduction des tracteurs et enjambeurs, imposée par les impératifs de productivité, a pu parfois aboutir à une banalisation, voire une dégradation des paysages.

Plusieurs phénomènes sont intervenus :

- Simplification du paysage par agrandissement des parcelles (remembrement)

- Restructuration des coteaux

- Elimination des composantes unitaires paysagères (arbres isolés ou alignés, haies, talus, bosquet) ou architecturales (croix, cabanes de vignes etc.).

La réorganisation d'un parcellaire viticole est un choix qui engage le viticulteur dans un projet à long terme. Les vignobles plantés sur de fortes pentes exposées au

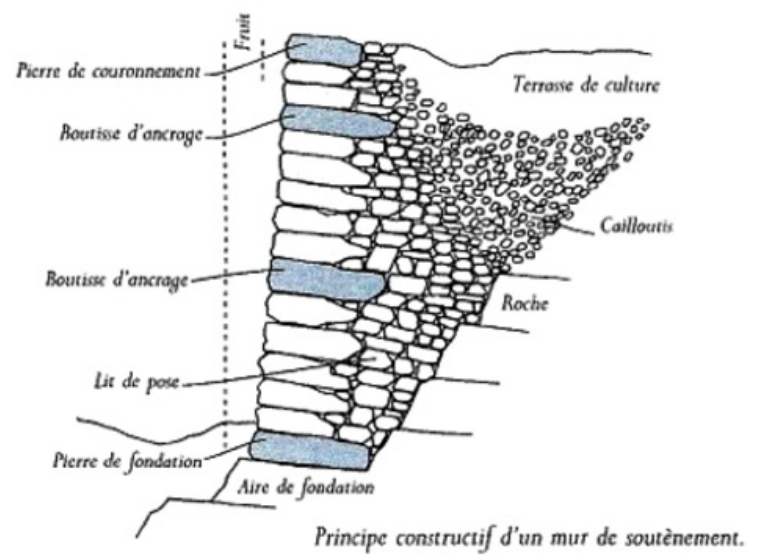

Figure 2. Coupe schématique d'un mur de soutènement, éléments de construction, extrait de «La pierre sèche, mode d'emploi » par Christian Lassure, éd. Eyrolles (2014).

sud et sud-est reçoivent les plus fortes radiations et sont les plus favorables à la maturation de la vigne. Cette particularité microclimatique, ainsi que l'accès à des terres moins coûteuses que celles des plaines, ont historiquement orienté le vigneron vers les zones de coteaux pour produire des vins de qualité. Pour pouvoir travailler le sol, il fallait empêcher le ruissellement, casser la pente afin de briser la course de l'eau et retenir une terre rare et fugitive. Derrière un mur de pierres sèches soigneusement choisies, les cailloux servent au drainage. Le tout retient une épaisse couche de terre (Fig. 2).

Ce savoir-faire historique témoigne de l'intelligence pragmatique et de la ténacité de nos ancêtres vignerons. C. Rossi et H. Filipetti soulignent ainsi que : « bien plus que celle des maisons elles-mêmes, la construction des murets et des terrasses mobilisa des forces considérables et mit en cuvre des volumes de pierres que l'on a peine à imaginer. Car en même temps qu'il épierrait ses champs pour pouvoir labourer, le paysan en garnissait le pourtour pour monter les murs qui en protégeraient l'accès, dessinant ainsi un maillage serré de chemins cernés de clôtures minérales, [...] avec, pour compléter le tout, un réseau d'escaliers et de marches à vous couper le souffle. [...] Un véritable monument à la ténacité des hommes pour survivre, à leur ingéniosité, à leur misère aussi $\gg[5]$.

Comme le précise R. Ambroise «les paysages de terrasses représentent le schéma le plus abouti de nature façonnée par l'homme. Formés d'une multitude d'oppositions, ils résonnent de tous leurs harmoniques, pour reprendre une comparaison musicale, et chacun de nous peut trouver, dans leur extrême complexité, l'espace qui répond à une aspiration en fonction de sa propre histoire, de sa culture, de son humeur à l'instant qui passe » (Fig. 3) [6].

La plupart de ces paysages de terroirs héroïques mériteraient une reconnaissance patrimoniale universelle mis en avant par J.F. Blanc : « Les terrasses peuvent être considérées comme un élément révélateur de l'évolution d'une civilisation paysanne et constituent ainsi un des exemples les plus significatifs des paysages culturels vivants européens $\gg[7]$.

Le coût d'exploitation des terrasses est généralement plus élevé qu'en plaine. Par ailleurs la modernité 


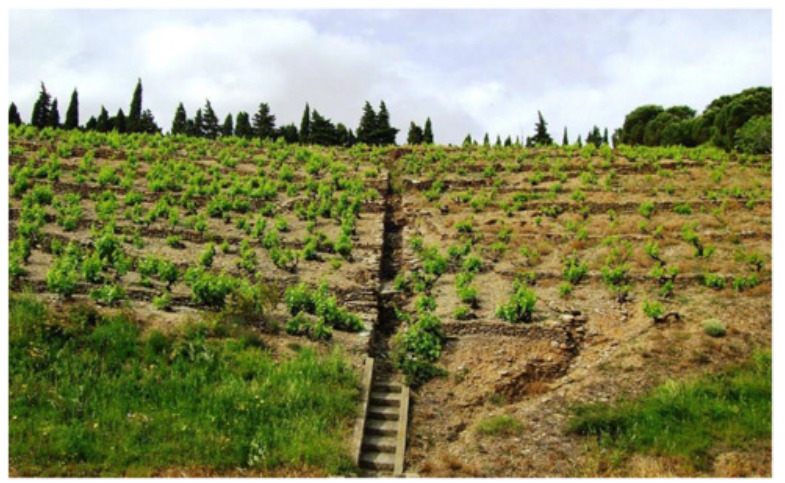

Figure 3. Le vignoble de Banyuls dans le Roussillon est implanté sur de très fortes pentes. Les vignes y sont aménagées en terrasses et entrecoupées d'un réseau de canaux destinés à évacuer efficacement l'eau lors des fortes pluies.

associée à la mécanisation de l'après-guerre à pénalisé ces territoires peu adaptés aux engins viticoles. A titre d'exemple, concernant la région de Valteline en Italie, D. Lorusso précise que «la grande période d'abandon du paysage en terrasses débute après la seconde Guerre Mondiale. En raison du boom industriel lié à la reconstruction, beaucoup des viticulteurs de Valteline quittent définitivement leurs vignobles pour s'installer dans les villes de la plaine du Pô, ou pour travailler dans les nouvelles usines créées au fond de la vallée. Certains vignobles sont alors convertis en d'autres cultures, tandis que beaucoup de terrasses sont abandonnées et laissées en friche, voire reboisées. Le premier recensement général de l'agriculture, en 1961, signale encore la présence de 3000 hectares de terrains plantés en vignes; néanmoins, trente ans après, lors du quatrième recensement, il n'en reste que 1700 ha. Le déclin se poursuit dans les décennies suivantes : les surfaces plantées en vignes atteignent à peu près 1100 ha en 2000 et se réduisent à 1000 ha en $2010 \gg$.

Par ailleurs ce handicap économique pourrait s'accentuer au cours des prochaines décennies avec le changement climatique (diminution du rendement par stress hydrique) et parallèlement la difficulté croissante de trouver du personnel acceptant la pénibilité du travail dans les terrasses.

En fonction du contexte local, la restauration de murets en pierre sèche, la création de banquettes, la gestion des talus, l'implantation d'arbres isolés ou de haies, peuvent s'intégrer dans un projet de création ou de restructuration des vignobles [8]. Parallèlement, une dimension esthétique, doit compléter l'approche fonctionnelle des aménagements hydrauliques (collecteurs, chemins, bassins ...).

L'aménagement des coteaux en terrasses était un fondement ancestral de la lutte contre l'érosion (Fig. 4). Malgré les contraintes et les coûts d'entretien des murs de soutien, il est parfois nécessaire de les privilégier dans les situations de forte pente (au-delà de $15 \%$ ). Les terrasses larges (20 à 50 mètres) imposent un remaniement poussé du coteau, qui facilite la mécanisation. Il présente cependant l'inconvénient du coût élevé et des risques de dévalorisation paysagère. Les terrasses étroites (1.80 à $2.30 \mathrm{~m}$ ) s'intègrent plus facilement dans le paysage. Selon

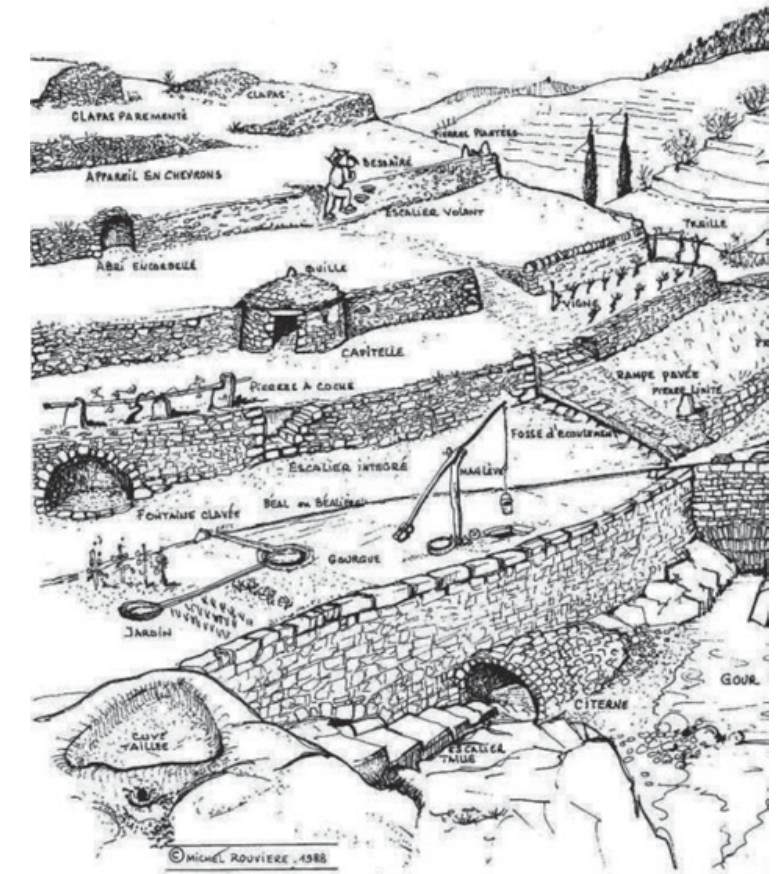

Figure 4. Les composantes des paysages de terrasses par M ROUVIERE, Source : paysages de terrasse, EDISUD (1993).
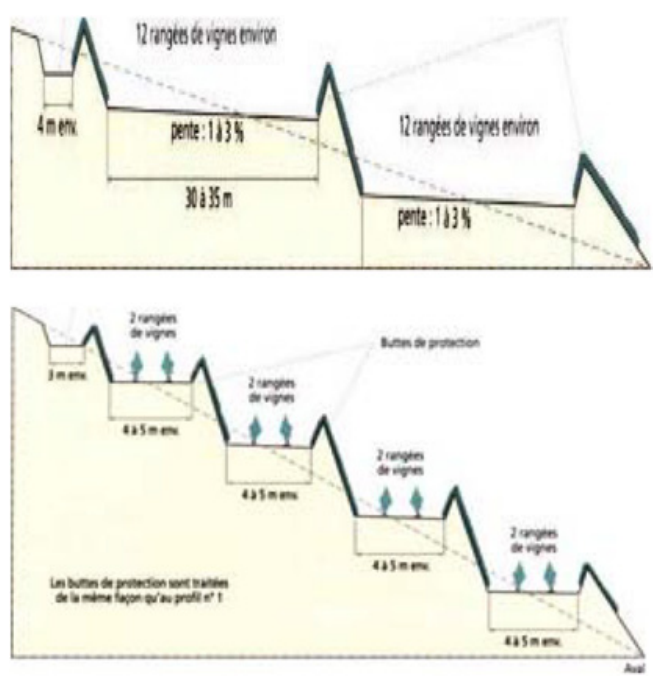

Figure 5. Exemple d'aménagement à Beaumes-de-Venise, $1^{\text {ère }}$ figure : banquette large, $2^{\text {ème }}$ figure : banquette, C. Milhaud, Aménagement et nature $\mathrm{n}^{\circ} 141$ (2001).

les cas, elles permettent d'implanter 1 ou 2 rangs par plateforme (Fig. 5).

\section{Biodiversité des vignobles héroïques}

Les murs de pierre sèche, qui constituent de précieux biotopes, forment de nombreuses cavités, refuges de la faune et la flore locales. Ces nombreuses niches accueillent une grande biodiversité. Certaines espèces jouent d'ailleurs le rôle important d'auxiliaire de culture, favorisant la protection ou la pollinisation des espèces végétales cultivées. Le GRABS du canton du Saint-Gall en Suisse précise ainsi que «avec leurs nombreuses fissures et la capacité de celles-ci de conserver la fraîcheur et d'emmagasiner la chaleur du soleil dans un environnement hostile, le mur de pierres sèches offre gîte et nourriture 


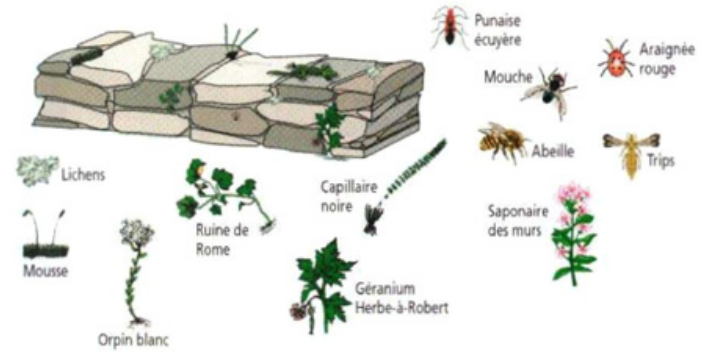

Figure 6. Rôle écologique d'un muret établi d'après le Guide Illustré de l'Écologie, B. Fischesser, M.F. Dupuis-Tate, Editions de la Martinière (2007).

à plusieurs plantes et animaux : algues, lichens et mousses, plantes de muraille comme la joubarbe, la rue des murailles, le cétérach officinal et la cymbalaire des murs, vers, escargots, araignées, mille-pattes, insectes, amphibies, reptiles et oiseaux, hérissons et martres. Des êtres vivants aux exigences les plus variées peuvent se sentir chez eux dans un mur de pierres sèches. Cette biodiversité est due aux différents microclimats qui se manifestent au cours de l'année à l'intérieur et aux parements du mur, inégalement exposés aux intempéries selon l'orientation. Tant pendant les hivers glacials qu'à la canicule, les fentes, fissures et trous des murs deviennent des quartiers de repos importants. Aux endroits surélevés, les murs servent de repères aux chasseurs ou de perchoirs aux passereaux. Ils favorisent en outre la diffusion et le maillage de la flore et de la faune en ce qu'ils constituent de bons points de passage entre les régions et les écosystèmes $\gg[9]$.

Le programme européen Biodivine (www . biodivine. eu) a permis d'établir les bases d'une vision fonctionnelle mais également naturalistes de la biodiversité dans les différents terroirs européens (France, Espagne et Portugal). C. Carlos souligne que l'Alto Douro Vinhateiro (ADV) est une zone classée au patrimoine mondial (UNESCO) de la région viticole du Douro, situé dans le nord-est du Portugal, dans le bassin du Douro. Cette région a plusieurs spécificités climatiques, géologiques, des sols et des variétés cultivées, qui confèrent des conditions uniques et singulières à la production de vins de Porto et du Douro. De plus, la présence d'une zone importante de vestiges patrimoniaux, de zones riveraines végétales ainsi que les terrasses de pierre sèches, peuvent contribuer à augmenter le réservoir de biodiversité dans cet agroécosystème viticole, le rendant unique pour le volet environnemental de la durabilité (Fig. 7).

Le projet LIFE AGROLIFE mené à Chypre, financé par la Commission européenne, avait pour but de redynamiser les vignobles traditionnels de Laneia et de Kapileio en s'appuyant notamment sur la biodiversité locale. Cette île méditerranéenne élabore le célèbre vin doux Commandaria issu des variétés locales mavro et xynisteri. Des murs de pierres sèches ont été créés ou restaurés pour limiter l'érosion et servir de refuge aux invertébrés et reptiles. Des bordures de haies traditionnelles ont été restaurées autour des vignobles pour préserver des espèces importantes de la faune locale. Des empilements de roches ont été créés pour servir d'habitat à des espèces importantes de reptiles et d'insectes (Fig. 8).

En France, un projet portant sur l'agroécologie a été développé par l'IFV et l'INAO afin d'intégrer des

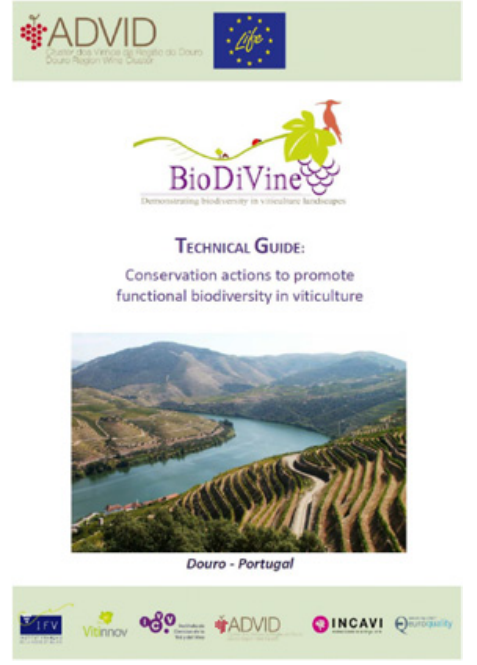

Figure 7. Guide technique de la biodiversité, établi dans la région du Douro au Portugal par l'ADVID, dans le cadre du projet européen Biodivine. www. biodivine.eu.

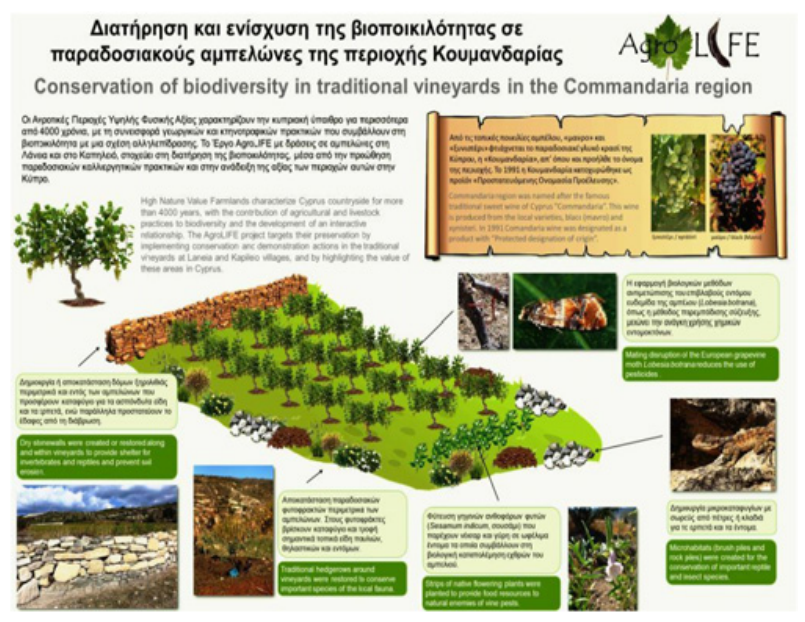

Figure 8. Projet LIFE Agrolife à Chypre. http:// agrolife.eu.

éléments de la biodiversité dans les cahiers des charges des appellations d'origine contrôlées et protégées [10].

Le guide précise notamment que «Les aménagements paysagers favorables à la biodiversité doivent être interconnectés dans l'espace selon une stratégie réfléchie à l'échelle paysagère. Tout en essayant de faire le lien entre les espaces semi-naturels déjà présents, ils doivent être orientés à proximité des espaces les plus défavorables à la biodiversité afin de constituer des zones refuges qui fournissent des abris et des ressources alimentaires à la faune. Pour cela, les espaces interparcellaires, doivent donc être maintenus et si possible renforcés.

L'agroécologie concerne tout particulièrement les terrasses et les banquettes, qui représentent d'excellents supports de biodiversité dans les vignobles de forte pente » (Fig. 9).

Parallèlement un outil pédagogique, propose la lecture paysage et la visualisation des orientations agroécologiques et des mesures agroenvironnementales par thématique, règlementaire ou en lien avec les cahiers des charges, pour un paysage-type. 


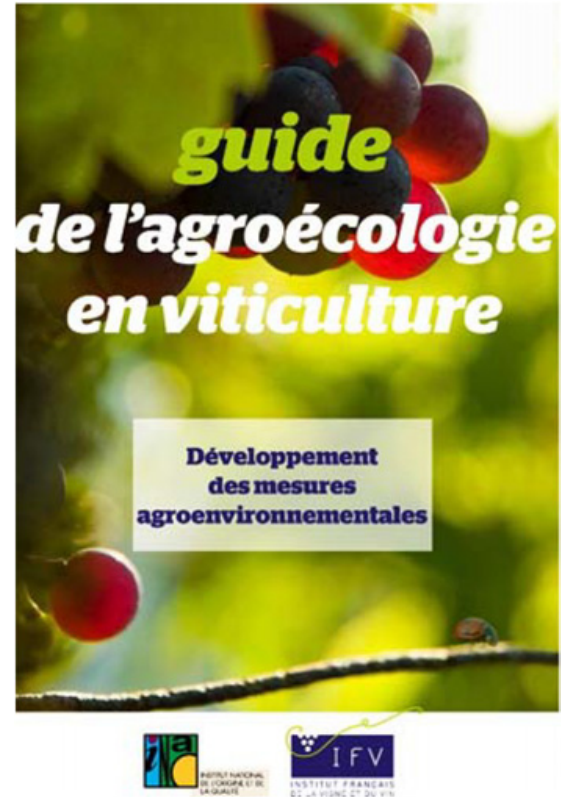

Figure 9. Guide de l'agroecologie en viticulture IFV/INAO http://www.vignevin.com/fileadmin/users/ifv/2015 New_Site/AE4_Territoire/Fichiers/GuideAgroEcologie -web.pdf.

\section{Conclusion}

La fonction strictement économique d'un territoire est de produire un bien qui est valorisé par sa commercialisation. Ainsi, les raisins sont transformés en vin, dont la vente conditionne l'équilibre financier d'une exploitation [11]. Mais l'intégration de la durabilité et du patrimoine dans l'économie associe également les effets territoriaux, sociaux et environnementaux de cette production, caractérisée par la notion d'externalité. Les externalités négatives peuvent éventuellement conduire à des coûts pour les citoyens ou les collectivités territoriales (en zone de coteaux, nécessité par exemple pour les collectivités, de gérer la terre qui a dévalé par ruissellement/érosion sur les routes et dans les villages). À l'inverse, selon les situations, les vignobles peuvent comporter des effets positifs sur les territoires (limitation des incendies, maintien d'une vie rurale) et parfois attractivité vis-à-vis du tourisme (paysage, patrimoine historique, etc.), non rémunérés par le prix de vente, à l'exception éventuellement d'un bénéfice « image » et d'une dynamique de vente directe [12].

Dans les régions méditerranéennes, qui seront probablement de plus en plus affectées par des incendies en lien avec le réchauffement climatique, les vignobles peuvent limiter l'impact des feux de forêt et de broussaille. Comme le souligne M. CONSTANS «Après le catastrophique incendie de 1986, on redécouvre le rôle pare-feu des vignes et l'on met en place une politique de relance de la vigne, notamment en créant de nouvelles vignes à l'interface des zones cultivées et des zones de forêt ou de maquis. En effet l'enjeu est considérable; d'une part la sécurité des personnes et des biens est concernée, d'autre part le développement touristique, qui est alors la grande priorité des acteurs économiques, ne peut faire bon ménage ni avec ce type de risque, ni avec les désolants paysages d'après l'incendie » (Fig. 10) [13].

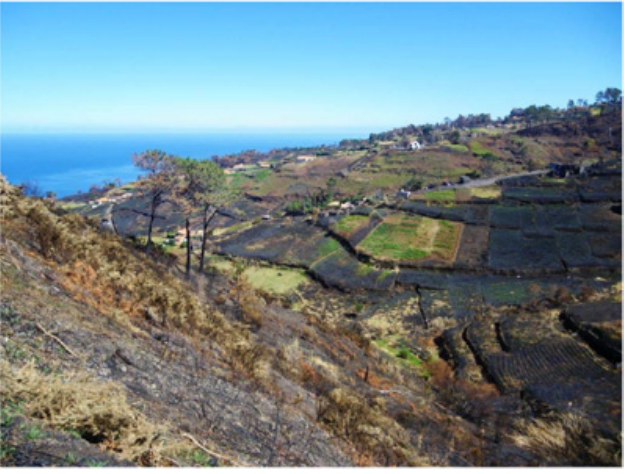

Figure 10. Paysage de Ponta do Pargo à l'ouest de l'île portugaise de Madère. En 2012, une bonne partie de l'ouest de l'île, avec de nombreuses parcelles de vignes, a subi un incendie très dévastateur.

Les terroirs viticoles et particulièrement les zones de forte pente contribuent à la richesse patrimoniale de nombreuses régions du monde. Mais les coûts de production des vignes de terrasse sont élevés, et celles-ci peuvent être abandonnées sans une politique volontariste des institutions régionales ou nationales. Cette approche justifie une politique d'aides pour ces terroirs, souvent 《héroïques 》, à forte valeur patrimoniale et écologique, si l'on ne veut pas qu'ils disparaissent à jamais [14].

Que soit remercié le CERVIM, qui assure la défensebreak et la promotion des vignobles héroïque et qui a organisé en 2018 un colloque sur l'île de Tenerife en Espagne, en lien avec la biodiversité. www. cervim.org.

\section{Références}

[1] J. Rochard, Bases de l'eco-oenotourisme: Du paysage à la Conception des Caves, CULTUR, ano $08-3$ - Outubro/, www.uesc.br/ revistas/culturaeturismo (2014)

[2] J. Rochard, Eco-construction et Développement durable, Revue des œnologues et des techniques vitivinicoles et œnologiques, ISSN 0760-9868 36, $\mathrm{N}^{\circ}$. Extra 133, pages 11-12 (2009)

[3] J. Rochard, Du paysage à l'Eco-conception des caves : « L'œnologue ambassadeur de l'ecooenotourisme $\gg$. Revue française d'œnologie, ISSN 0395-899X 252, pages. 4-7 (2012)

[4] S. Michel, Assemblée Générale de l'AVC, Le vigneron Champenois (2000)

[5] C. Rossi et H. Fillipetti, Le patrimoine rural français, Paris, Eyrolles (2007)

[6] R. Ambroise, P. Fripa, S. Giorgis, Paysages de terrasses, Edisud (1995)

[7] J.F. Blanc, Terrasses d'Ardèche, Paysages et patrimoine, ouvrage à compte d'auteur (2001)

[8] D. Lorusso, Culture de la vigne, production et commerce du vin de Valteline (XIXe-XXe siècles) : Valorisation qualitative et crise du paysage viticole traditionnel, Territoires du vin $\mathrm{n}^{\circ} 6$ - Territoires du vin d'Italie, Disponible sur Internet: http:// revuesshs.u-bourgogne.fr/territoiresduvin/ document.php?id=1892 ISSN 1760-5296 (2014) 
[9] Grabs, Construction en pierres sèches Office Fédéral de la culture suisse (2012)

[10] J. Rochard, C. Herbin, V. Lempereur, L'agroécologie des terroirs, Concept, application viticole, exemple de la France, Revue des Enologues, 166, janvier (2018)

[11] M. Constans, Le patrimoine paysager viticole de Banyuls entre reconstruction et destruction. In : Rencontres du Clos-Vougeot (2009) - Patrimoines et paysages viticoles. Chaire UNESCO Culture et tradition du vin, ISBN 978-2-918173-09-0 (2010)
[12] J. Rochard, Traité de viticulture et d'oenologie durables (2005), Editions Avenir Enologie. Grabs, Construction en pierres sèches, canton du Saint-Gall (2010)

[13] J. Rochard, Vignes et Terroir, Splendeur des Paysages du Monde, Editions La France agricole (2017)

[14] J. Rochard, Contribution of the Biodiversity to the Eco-Winetourism of the Heroic Vineyards: Assets and Prospects. 6 th international congress on mountain and steep slope viticulture, La Laguna Tenerife Island, Spain (2018) 